\title{
Monitoring and Prevention the Risk of Diabetic Foot Ulcer Infection during Coronavirus Disease-19 Pandemic: A Narrative Review and Perspective Algorithm
}

\author{
Mariya V. Dmitriyeva ${ }^{1}$ D, Saken K. Kozhakhmetov ${ }^{1}$ (D), Dulat K. Turebayev ${ }^{1}$ (D), Saltanat N. Urazova ${ }^{1}$, Talgat M. Omarov ${ }^{1}$, \\ Nurbek S. Igissinov ${ }^{1,3}$, Medet T. Toleubayev ${ }^{1,2 *}$ (D) \\ ${ }^{1}$ Department of Public Health, Astana Medical University, Nur-Sultan, Kazakhstan; ${ }^{2}$ Department of Surgery, Multidisciplinary \\ City Hospital No. 1, Nur-Sultan, Kazakhstan; ${ }^{3}$ Central Asian Cancer Institute, Nur-Sultan, Kazakhstan
}

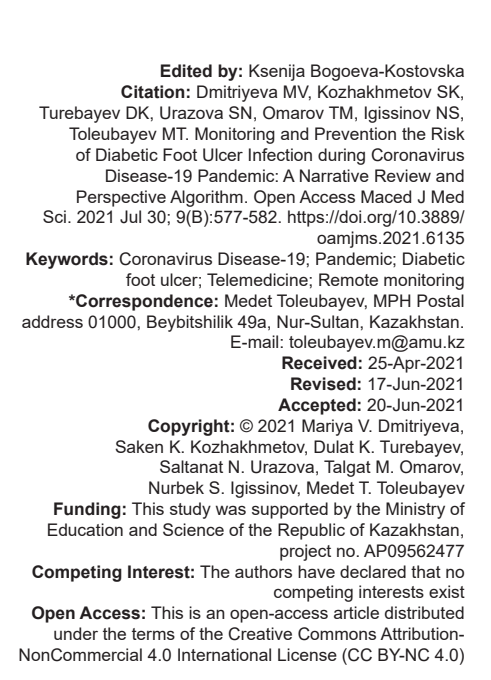

\section{Abstract}

BACKGROUND: The coronavirus disease (COVID)-19 pandemic leads to significant changes in the healthcare system and undermining best practices for maintaining a diabetic limb. A large number of patients with diabetic foot are left without timely medical care and are at increased risk of complications, hospitalization, lower limb amputation, and death. A new paradigm must be adopted for the transition from inpatient care to community-based care. The introduction of a pandemic remote management for patients with diabetic foot ulcer includes an assessment of the risk of complications through telemedicine and further stratification of patients according to the developed algorithm.

METHODS: A literature review was performed for articles related to telemedicine. We used PubMed, Google Scholar Cochrane Library, and Ovid MEDLINE to search published articles. We used the following keywords: "Telemedicine," "diabetes mellitus," "COVID-19," "diabetic foot ulcer," and "remote monitoring."

RESULTS: Implementation of the proposed pandemic care includes telemedicine for remote monitoring and treatment of patients with diabetic foot ulcers, as well as an algorithm for determining the risk of diabetic ulcer infection and patient management tactics according to the identified risk.

CONCLUSION: The management of patients with diabetic foot ulcers during a pandemic includes the following goals - to reduce the burden on the health-care system, maintain the safety and functionality of diabetic foot at home, and reduce the risk of COVID-19 in patients with diabetic foot ulcers.

\section{Introduction}

Most patients with diabetic foot ulcers need a health-care professional regularly to receive adequate wound care [1]. Delays in the treatment of diabetic foot ulcers associated with limited outpatient doctor visits during a pandemic can lead to complications, which increases the risk of emergency hospitalizations, lower limb amputation and imposes an additional burden on the health-care system during the ongoing crisis and leads to severe consequences for patients [2], [3], [4]. Moreover, patients with diabetes mellitus and diabetic foot syndrome often have multiple comorbidities and meet several criteria for a high risk of coronavirus infection [5]. However, limiting physician visits and planned hospitalizations are essential to reduce the risk of infection in the coronavirus disease (COVID)19 era.
The pandemic crisis has brought about significant changes in the management of patients with chronic diseases around the world [6]. Traditional outpatient care focused on regular monitoring of complications had to be replaced by untested telemedicine use and patient self-reporting.

The proposed algorithm for telemedicine management of patients with diabetic foot ulcers to reduce the risk of ulcer infection during a pandemic may be important for combating the triple threat - the risk of diabetic foot ulcer infection, the risk of an increase in the number of emergency hospitalizations, and the risk of COVID-19 infection.

The pandemic reorganization of health-care systems to contain the COVID-19 emergency has changed standard practice, leaving a large number of patients without forehanded care, including those with diabetic foot ulcers [7]. Massive quarantines, restrictions on elderly people leaving their homes, and reduced 
public transport can lead to serious consequences, such as an increased risk of complications of diabetic ulcers and an increased risk of lower limb amputation and death [8]. Insufficient observation and untimely treatment of superficial diabetic ulcers during restrictive quarantine measures can lead to infection of the ulcer, the need for emergency hospitalization, which, in turn, may contribute to a more rapid spread of the pandemic [9]. Diabetic ulcer infection can be prevented and controlled with regular check-ups and early treatment [10].

The COVID-19 pandemic has brought virtual care technology to the fore, given the important and ongoing progress made in this area [11]. These technologies provide the initial needed assistance, protecting vulnerable people and preventing the spread of disease, given the physical distance [12]. Traditional care of patients with diabetic foot ulcers requires faceto-face contact for procedures (wound debridement), although virtual nursing technologies may also offer useful telecommuting capabilities for these patients under the supervision of a physician [13].

Patients of their family, with the help of remote consultations of general practitioners, can assess the possible presence of wound infection by the appearance of the wound, body temperature, and heart and respiratory rate [14]. Use two-way video and photo sharing FaceTime, W App for wound assessment, a combination of save and forward photos, Zoom Short Message Service or text video chat, and mobile wound area measurement applications (such as the free imitoMeasure mobile app) can assist the physician in visual assessment of wounds (detection of infection and assessment of the progress of wound healing) and the appointment of appropriate treatment [15] (Figure 1).

Then, also through video communication, a mobile application, and instant messengers, patients and their doctors can track the effectiveness of treatment and the speed of healing of a diabetic ulcer.

The characteristics of medicines and medical supplies and the correct choice of a doctor are of paramount importance for providing homecare during a pandemic. For example, antibiotic therapy in oral forms, disposable wipes with disinfectant solutions for wound care, the use of readily available, inexpensive and easyto-use dressings, and wound dressings with detailed instructions and video tutorials on how to use drugs or change dressings are innovative ways that can be used for remote observation and treatment of patients with diabetic foot ulcers during a pandemic [16].

Thus, the use of telemedicine can be very useful for sorting patients according to the degree of risk of diabetic ulcer infection, remote management, and selection of appropriate care [17]. However, there has never been widespread adoption of telemedicine in the management of patients with diabetic ulcers, and the short- and long-term results of the efficacy of remote monitoring and treatment are unknown [18]. Although a remote diabetic ulcer scoring system may not be able to fully and accurately assess the risk of complications, the system is relatively simple and reliable for determining the size of a diabetic ulcer and signs of infection, especially given the second wave of the pandemic, COVID-19 [19].

There is an urgent need to develop, implement, and approve structured algorithms for the care of this category of patients based on telemedicine for their
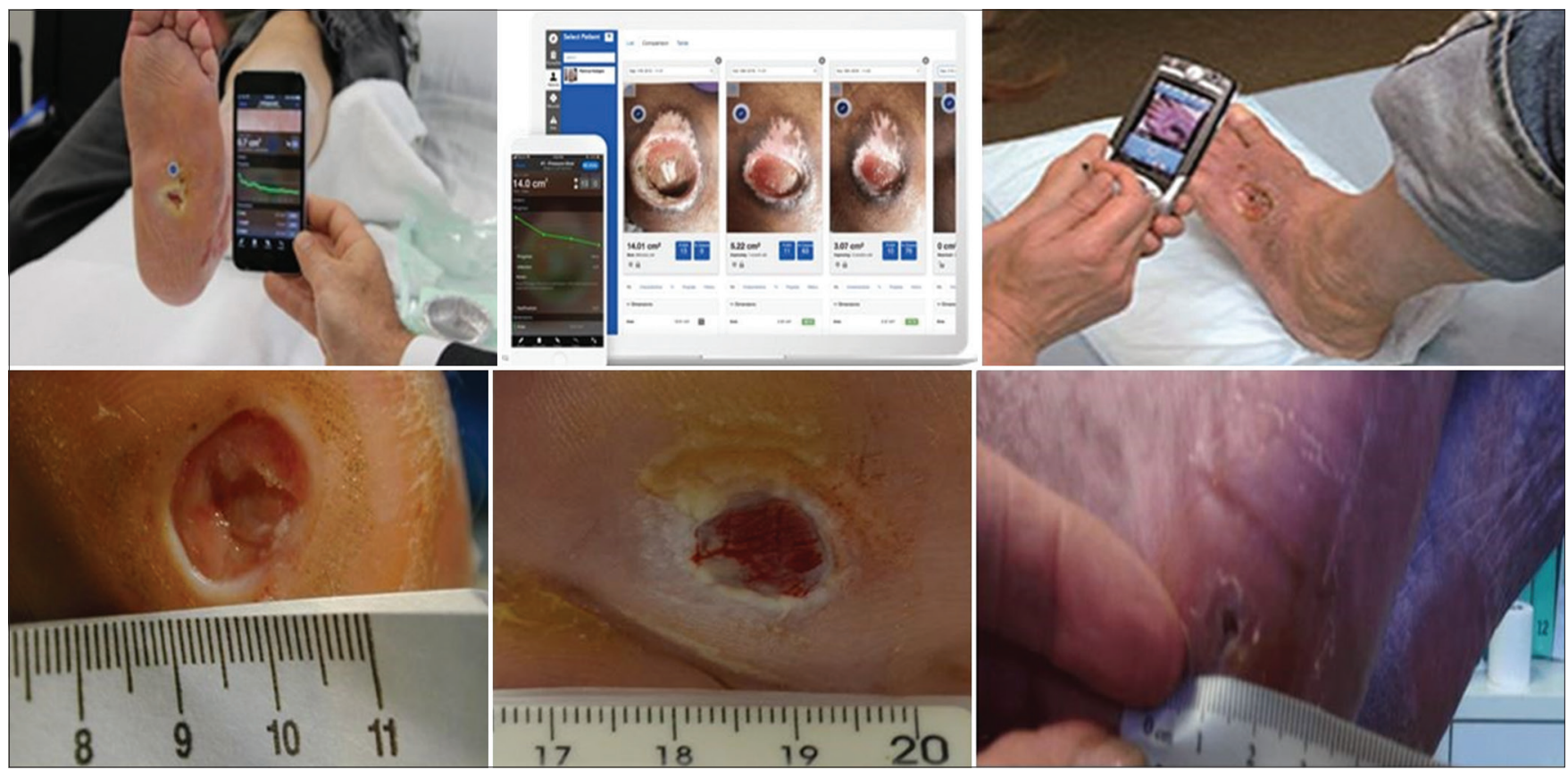

Figure 1: Measuring the area of a diabetic ulcer by a patient using a mobile application, a photo taken by a patient of a diabetic ulcer to assess the effectiveness of treatment and the risk of complications 
treatment and prevention of complications of diabetic foot syndrome [20].

\section{Methods}

A literature review was performed for articles related to telemedicine. We used PubMed, Google Scholar, Cochrane Library, and Ovid MEDLINE to search published articles. We used the following keywords: "Telemedicine," "diabetes mellitus," "COVID19 ," "diabetic foot ulcer," and "remote monitoring."

\section{Results}

The COVID-19 pandemic requires a rapid change in best practices for health-care delivery and the implementation of new and effective protocols to reduce the development of complications of chronic nonCOVID-19 diseases. New clinical needs, dictated by the pandemic, were a prerequisite for the development of a pandemic algorithm for the care of patients with diabetic foot ulcers.

The developed algorithm for accelerated tracking of the risk of diabetic ulcer infection to reduce the number of cases of late referral to specialists, and to avoid hospitalization in stable clinical cases to reduce the risk of coronavirus infection. The proposed algorithm for managing patients with diabetic ulcers to reduce the risk of ulcer infection during the COVID-19 pandemic is shown in Figure 2.

The proposed algorithm is aimed at sorting patients according to the risk of diabetic foot ulcer infection at the telemonitoring level (low, medium, high, and urgent) and related further methods of care.

It is also possible to track the results of treatment and changes in the size and depth of a diabetic ulcer overtime using remote consultations and using mobile applications and messengers every 2 weeks in the absence of signs of infection, every week with a low risk of infection. In particular, photos of diabetic ulcers and measurements using the mobile app should be updated each time the dressing is changed to facilitate changes in the homecare strategy according to the wound status.

If a high risk of infection with a diabetic ulcer is identified, an outpatient examination may be recommended, subject to all anti-epidemic measures, for examination, surgical treatment of a diabetic ulcer and prescribing additional treatment. Further, it is possible to return to remote inspection with control every 3-5 days. If a high risk is identified, the progression of infection and the appearance of signs of alertness during a remote examination, such as the presence of infection of a diabetic foot ulcer (hyperemia around the ulcer, cellulite, pus secretion, edema, pain, and local temperature increase), and deterioration in dynamics (increase the size of the ulcer, damage to soft tissues/ bones, and increasing signs of ischemia or infection), it is necessary to resolve the issue of emergency hospitalization.

Thus, based on the results of remote examination, patients with a low risk of infection with diabetic ulcers can be given recommendations for daily wound care and dressings on their own or by relatives under online supervision and medical supervision. A home call from a local general practitioner may also be available for suspected diabetic ulcer infection, if a low to moderate risk of infection is identified. With positive dynamics, short-term oral (1-2 weeks) antibiotic therapy continued sanitation of the diabetic ulcer and dressing at home can be recommended. The identification of a high risk, progression of infection of a diabetic ulcer, is clinically significant enough to make a decision on the transition from remote examination to full-time reception in a polyclinic or making a decision on the need for emergency hospitalization.

\section{Discussion}

Diabetic foot ulcer is a serious chronic condition resulting from complications of diabetes. Approximately $10-15 \%$ of diabetic patients develop foot ulcers, and more than half of diabetic ulcers become infected [14], [21]. Furthermore, complications of diabetic foot ulcers are the most common cause of hospitalization associated with diabetes [22]. The incidence of lower limb amputation in patients with high and urgent risk of diabetic ulcer is approximately $20 \%$ [14]

At present, the 2019 COVID-19 pandemic is likely to pose a threat to medical care, with some unintended consequences [23]. Cancellation of most outpatient clinical services poses a potential threat to patients with diabetic foot ulcers, since significant delays in treatment increase the risk of ulcer infection and a poor prognosis [24], [25]. Late referral to specialists is still a common problem worldwide, and remote observation and timely treatment of diabetic foot ulcers increase the chances of recovery and reduce the frequency of lower limb amputations [26], [27], [28].

The current global situation dictates the need for the introduction of remote sensing technologies, as well as the development and implementation of effective measures for the provision of pandemic assistance to identify patients at high risk of complications of diabetic foot syndrome and protect them from infection with COVID-19. Traditional care of patients with diabetic ulcers requires personal contact during procedures 


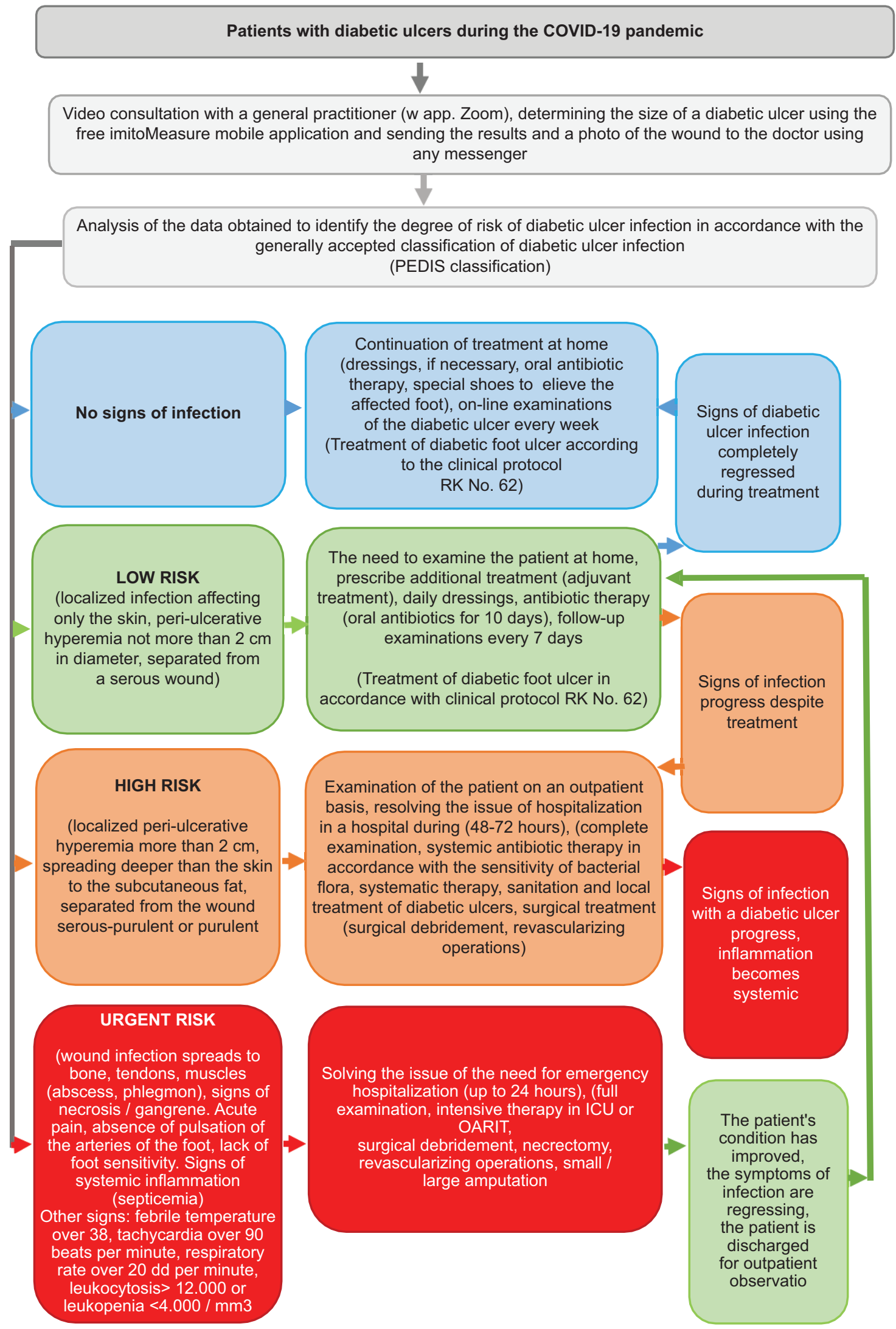

Figure 2: Algorithm for the management of patients with diabetic ulcers to reduce the risk of ulcer infection during the Covid-19 pandemic.

(wound debridement), virtual care technologies can be offered and applied to these patients during a pandemic. While there was no need for widespread adoption of telemedicine before the pandemic, the COVID-19 pandemic has brought virtual care technology to the fore, given the important and ongoing progress made in this area [29]. These technologies provide initial needed care, protecting vulnerable people and preventing the spread of disease, given physical distancing, and the short- and long-term results of reducing 
the risk of infection with diabetic ulcers are not yet known [30], [31]. At the same time, it is recommended to give priority attention to patients with a critical or high risk of diabetic ulcer infection to receive timely inpatient care, and patients with low and medium risk - through telemedicine and home visits [32]. Telemedicine management of diabetic ulcers can reduce resource use, improve patient care, and reduce the burden on health care during a pandemic [33].

Thus, the outbreak of COVID-19 is negatively affecting the provision of medical care for patients with diabetes. With the ongoing pandemic, massive efforts are still needed to mitigate the impact of COVID-19 on patients with diabetic foot ulcers.

In the current review, there are some limitations. This review was performed as a narrative and not a systematic review of the literature. Moreover, the proposed algorithm implementation needs in clinical practice to assess its efficiency.

\section{Conclusion}

The proposed algorithm combined the use of virtual technologies for monitoring and caring for diabetic foot ulcers, the ability to sort all patients into risk groups for diabetic ulcers infection, effective provision of personalized care based on the identified risk, which will lead to a decrease in the risk of complications, possible cases of late referral to specialists, and the risk of infection COVID-19 in a hospital, which in turn will reduce the burden on the health-care system in a pandemic crisis. The need for new and effective protocols to reduce the impact of non-COVID-19 disease on the burden of hospitalization is greater than ever, and implementing a diabetic ulcer patient management algorithm to reduce the risk of ulcer infection during the COVID-19 pandemic.

\section{References}

1. Armstrong DG. Boulton AJ, Bus SA. Diabetic foot ulcers and their recurrence. N Engl J Med. 2017;376:2367e75. PMid:28614678

2. Wise J. Early referral for foot ulcers is vital, finds audit of diabetes care. BMJ. 2016;352:i1820. https://doi.org/10.1136/ bmj. 11820 PMid:27034450

3. Faglia E, Clerici G, Caminiti M, Quarantiello A, Gino M, Morabito A. The role of early surgical debridement and revascularization in patients with diabetes and deep foot space abscess: Retrospective review of 106 patients with diabetes. J Foot Ankle Surg. 2006;45(4):220-6. https://doi.org/10.1053/j. jfas.2006.04.002

\section{PMid:16818148}

4. Rogers LC, Lavery LA, Joseph WS, Armstrong DG. All feet on deck-the role of podiatry during the COVID-19 pandemic: Preventing hospitalizations in an overburdened healthcare system, reducing amputation and death in people with diabetes. J Am Podiatr Med Assoc. 2020;2020:51. https://doi. org/10.7547/20-051

PMid:32208983

5. World Health Organization. WHO Coronavirus Disease (COVID19) Dashboard. Geneva: World Health Organization; 2020.

6. Rosenbaum $L$. The untold toll-the pandemic's effects on patients without Covid-19. N Engl J Med. 2020;382(24):2368-71. https:// doi.org/10.1056/nejmms2009984

PMid:32302076

7. Onder G, Rezza G, Brusaferro S. Case-fatality rate and characteristics of patients dying in relation to COVID-19 in Italy. J Am Med Assoc. 2020;323(18):1775-6. https://doi.org/10.1001/ jama.2020.4683

PMid:32203977

8. Guo W, Li M, Dong Y, Zhou H, Zhang Z, Tian C, et al. Diabetes is a risk factor for the progression and prognosis of COVID19. Diabetes Metab Res Rev. 2020;2020:e3319. https://doi. org/10.1002/dmrr.3319

PMid:32233013

9. Meloni M, Izzo V, Giurato L, Gandini R, Uccioli L. Management of diabetic persons with foot ulceration during COVID-19 health care emergency: Effectiveness of a new triage pathway. Diabetes Res Clin Pract. 2020;165:108245. https://doi. org/10.1016/j.diabres.2020.108245

PMid:32497745

10. Ruan Q, Yang K, Wang W, Jiang L, Song J. Clinical predictors of mortality due to COVID-19 based on an analysis of data of 150 patients from Wuhan, China. Intensive Care Med. 2020;46(5):846-8. https://doi.org/10.1007/s00134-020-05991-x PMid:32125452

11. Dhivya S, Padma VV, Santhini E. Wound dressings a review. Biomedicine (Taipei). 2015;5(4):22. https://doi.org/10.7603/ s40681-015-0022-9

PMid:26615539

12. COVID-19 and Diabetic Foot Disease. IWGDF Guidelines; 2020. Available from: https://iwgdfguidelines.org/covid-19. [Last accessed on 2020 Jun 08].

13. Papanas N, Papachristou S. COVID-19 and diabetic foot: will the lamp burn bright? The Int $\mathrm{J}$ Lower Extrem Wounds. 2020;19(2):111. https://doi.org/10.1177/1534734620921382 PMid:32320324

14. Lipsky BA, Berendt AR, Cornia PB, Pile JC, Peters EJ, Armstrong DG, et al. 2012 infectious diseases society of America clinical practice guideline for the diagnosis and treatment of diabetic foot infections. Clin Infect Dis. 2012;54(12):e132-73. https://doi.org/10.1093/cid/cis346 PMid:22619242

15. Armstrong DG, Rankin TM, Giovinco NA, Mills JL, Matsuoka Y A heads-up display for diabetic limb salvage surgery: A view through the google looking glass. J Diabetes Sci Technol. 2014;8(5):951-6. https://doi.org/10.1177/1932296814535561 PMid:24876445

16. Kelahmetoglu O, Camlı MF, Kirazoglu A, Erbayat Y, Asgarzade S, Durgun $\mathrm{U}$, et al. Recommendations formanagement of diabetic foot ulcers during COVID-19 outbreak. Int Wound J. 2020;17(5):1424-7. https://doi.org/10.1111/iwj.13416 PMid:32501604

17. Available from: https://www.mohfw.gov.in/pdf/Telemedicine.pdf [Last accessed on 2020 Jun 08]. 
18. Ghosh A, Gupta R, Misra A. Telemedicine for diabetes care in India during COVID-19 pandemic and national lockdown period: Guidelines for physicians. Diabetes Metab Syndr. 2020;14(4):273-6. https://doi.org/10.1016/j.dsx.2020.04.001 PMid:32283497

19. Chuan F, Tang K, Jiang P, Zhou B, He X. Reliability and validity of the perfusion, extent, depth, infection and sensation (PEDIS) classification system and score in patients with diabetic foot ulcer. PLoS One. 2015;10(4):e0124739. https://doi.org/10.1371/ journal.pone. 0124739

PMid:25875097

20. Diabetes Foot Care in the COVID-19 Pandemic. Available from: https://www.d-foot.org/resources/columns/feet-first/ diabetes-foot-care-in-the-covid-19-pandemic. [Last accessed on 2020 Jun 08]. https://doi.org/10.2337/dc20-1176

21. Prompers L, Huijberts $M$, Apelqvist J, Jude E, Piaggesi $A$ Bakker $\mathrm{K}$, et al. High prevalence of ischaemia, infection and serious comorbidity in patients with diabetic foot disease in Europe: Baseline results from the Eurodiale study. Diabetologia. 2007;50(1):18-25. https://doi.org/10.1007/ s00125-006-0491-1

PMid:17093942

22. Skrepnek GH, Mills JL Sr., Lavery LA, Armstrong DG. Health care service and outcomes among an estimated 6.7 million ambulatory care diabetic foot cases in the U.S. Diabetes Care. 2017;40(7):936-42. https://doi.org/10.2337/dc16-2189 PMid:28495903

23. Ma RC, Holt RI. COVID-19 and diabetes. Diabet Med. 2020;37(5):723-5. PMid:32242990

24. Hussain A, Bhowmik B, do Vale Moreira NC. COVID-19 and diabetes: Knowledge in progress. Diab Res Clin Pract. 2020;162:108142. https://doi.org/10.1016/j. diabres.2020.108142 PMid:32278764

25. Bornstein SR, Rubino F, Khunti K, Mingrone G, Hopkins D, Birkenfeld $\mathrm{AL}$, et al. Practical recommendations for the management of diabetes in patients with COVID-19. Lancet
Diab Endocrinol. 2020;8(6):546-50.

PMid:32334646

26. Manu C, Lacopi E, Bouillet B, Vouillarmet J, Ahluwalia R, Lüdemann $\mathrm{C}$, et al. Delayed referral of patients with diabetic foot ulcers across Europe: Patterns between primary care and specialised units. J Wound Care. 2018;27(3):186-92. https://doi. org/10.12968/jowc.2018.27.3.186

\section{PMid:29509115}

27. Smith-Strwm H, Iversen MM, Igland J, Østbye T, Graue M, Skeie $S$, et al. Severity and duration of diabetic foot ulcer (DFU) before seeking care as predictors of healing time: A retrospective cohort study. PLoS One. 2017;12(5):e0177176. https://doi.org/10.1371/journal.pone.0177176

PMid:28498862

28. Tao F, Tang X, Tao H, Luo Y, Cao H, Xiang W, et al. Surgical treatment of diabetic foot ulcers during the COVID-19 pandemic in China. J Diabetes Complicat. 2020;34(9):107622. https://doi. org/10.1016/j.jdiacomp.2020.107622

PMid:33051005

29. Armstrong DG, Giovinco N, Mills JL, Rogers LC. Facetime for physicians: Using Real time mobile phone-based videoconferencing to augment diagnosis and care in telemedicine. Eplasty. 2011;11:e23.

PMid:21559249

30. Office for Civil Rights (OCR). Notification of Enforcement Discretion for Telehealth; 2020. Available from: https://www. hhs.gov/hipaa/for-professionals/special-topics/emergencypreparedness/notification-enforcement-discretion-telehealth/ index.html. [Last accessed on 2020 Mar 21].

31. Armstrong DG, Rowe VL, D'Huyvetter K, Sherman RA. Telehealth-guided home-based maggot debridement therapy for chronic complex wounds: Peri and post-pandemic potential. Int Wound J. 2020;17(5):1490-5. https://doi.org/10.1111/iwj.13425 PMid:32558362

32. Roser M, Ritchie H, Ortiz-Ospina E, Hasell J. Coronavirus Pandemic (COVID-19). Our World in Data; 2020. Available from: https://www.ourworldindata.org/coronavirus. [Last accessed on 2020 Mar 21]. 